\title{
Using network analysis to identify seasonal patterns and key nodes for risk-based surveillance of pig diseases in Italy
}

\author{
Maria Ines Crescio ${ }^{1}$ (D) | Gianluca Mastrantonio ${ }^{2}$ | Silvia Bertolini ${ }^{1}$ | Cristiana Maurella ${ }^{1}$ | \\ Amie Adkin $^{3}$ | Francesco Ingravalle ${ }^{1}$ | Robin R. L. Simons ${ }^{4}$ | Marco DeNardi ${ }^{5}$ (D) | \\ Katharina Stark ${ }^{5}$ | Agustin Estrada-Peña ${ }^{6}$ | Giuseppe Ru ${ }^{1}$
}

${ }^{1}$ Istituto Zooprofilattico Sperimentale di Piemonte, Liguria e Valle d'Aosta (IZSTO), Torino, Italy

${ }^{2}$ Dipartimento di Scienze Matematiche, Politecnico di Torino, Torino, Italy

${ }^{3}$ Foods Standards Agency, London, UK

${ }^{4}$ Animal and Plant Health Agency (APHA), Addlestone, UK

${ }^{5}$ SAFOSO AG, Liebefeld, UK

${ }^{6}$ University of Zaragoza (UNIZAR), Zaragoza, Spain

\section{Correspondence}

Maria Ines Crescio, Istituto Zooprofilattico Sperimentale del Piemonte, Liguria e Valle d'Aosta (IZSPLVA), Via Bologna 148, 10154 Torino, Italy.

Email: mariaines.crescio@izsto.it

Gianluca Mastrantonio, Department of Mathematical Sciences - Politecnico di Torino, Corso Duca degli Abruzzi, 24 - 10129 Torino, Italy.

Email: gianluca.mastrantonio@polito.it

\section{Funding information}

This work had funding agreed through the Animal Health and Welfare ERANETconsortium (https://www.anihwa. eu/) under SPARE ('Spatial risk assessment framework for assessing exotic disease incursion and spread through Europe'). Funders are acknowledged as the Department for the Environment, Food and Rural Affairs (Defra) - UK, Ministry of Health - Italy, Spanish National Institute of Agriculture and Food Research and Technology - Spain, and Federal Food Safety and Veterinary Office (FSVO) Switzerland.

\begin{abstract}
The description of the pattern of livestock movements between herds provides essential information for both improving risk-based surveillance and to understand the likely spread of infectious diseases. This study provides a description of the temporal pattern of pig movements recorded in Italy on a 4-year period (2013-2016). Data, provided by the National Livestock registry, were described by social network analysis and the application of a walk-trap algorithm for community detection. Our results show a highly populated community located in Northern Italy, which is the focal point of the Italian industrial pig production and as a general pattern an overall decline of medium and backyard farms and an increase in the number of large farms, in agreement with the trend observed by other EU pig-producing countries. A seasonal pattern of all the parameters evaluated, including the number of active nodes in both the intensive and smaller production systems, emerged: that is characterized by a higher number of movements in spring and autumn, linked with the breeding and production cycle as pigs moved from the growing to the finishing phase and with periods of increased slaughtering at Christmas and Easter. The same pattern was found when restricting the analysis to imported pig batches. Outbreaks occurring during these periods would have a greater impact on the spread of infectious diseases; therefore, targeted surveillance may be appropriate. Finally, potential super-spreader nodes have been identified and represent $0.47 \%$ of the total number of pig holdings $(n=477)$. Those nodes are present during the whole study period with a similar ranking in their potential of being super-spreaders. Most of them were in Northern Italy, but super-spreaders with high mean out-degree centrality were also located in other Regions. Seasonality, communities and super-spreaders should be considered when planning surveillance activity and when applying disease control strategies.
\end{abstract}

\section{KEYWORDS}

movements, pigs, social network analysis, surveillance, swine, time series 


\section{1 | INTRODUCTION}

Social network analysis (SNA) is a technique initially developed in sociology to investigate the links between local patterns of relationships. In an epidemiological setting, the description of a social structure provides a flexible framework for investigating associations or interactions and, consequently, helps in addressing a broad set of questions. An advantage of the analytical approach of SNA, compared to other techniques, is the ability to handle bi-directional relationships within groups such as contacts between individuals, trade or animal movements (Dube et al., 2011; Martinez-Lopez et al., 2009b). Following the increasing availability of livestock movement data, SNA has been extensively applied in veterinary science to assess infectious disease dynamics.

A network is a tool, used in many scientific fields, to visualize and understand relationship/connections between 'objects' of interest. Those objects belong to a variety of fields, going from people in social sciences to molecules in chemistry.

In veterinary science the unit of interest (node) is either the animal or the holding in terms of farm or staging point or collecting centre and the movements represent the relationship (tie), each node and each tie can be associated to one or more attributes (e.g. the farm code, the number of heads per farm), therefore SNA can be a useful surveillance tool by helping to identify holdings central in the flow of livestock production that can be targeted in order to accelerate the detection of highly infectious diseases, or helping to understand the spread of an infectious disease during the period between introduction and first detection of the infectious agent (Dube et al., 2008; Martinez-Lopez et al., 2009b).

Movements of livestock such as cattle (Bigras-Poulin et al., 2004; Brennan et al., 2008; Frossling et al., 2012; Noremark et al., 2011; Rautureau et al., 2011; Vernon \& Keeling, 2009;), sheep (Kiss et al., 2006b; Volkova et al., 2010), poultry (Brioudes \& Gummow, 2017) and pigs (Bigras-Poulin et al., 2004; Brioudes \& Gummow, 2017; Buttner et al., 2013; Dorjee et al., 2013; Guinat et al., 2016; Lentz et al., 2011; Lindstrom et al., 2012; Noremark et al., 2011; Ribbens et al., 2009; Salines et al., 2017; Thakur et al., 2016) have already been described and modelled by SNA in several countries, using either movements recorded in databases or reported by farmers through questionnaires. In Italy, cattle movements have been extensively analysed by SNA with a focus on surveillance optimization (Bajardi et al., 2011, 2012; Natale et al., 2009), whereas, to our knowledge, there is no systematic characterization of pigs movements in Italy among the current literature, with the exception of a comparison among the spatial and functional organization of the pig trade in 2011 in four European Member States (EU MSs) (Relun et al., 2016). Italy is the seventh largest pork meat producer among EU MSs (http://ec.europa.eu/ eurostat/web/products-eurostat-news/-/DDN-20170919-1). The approximately 8.5 million pigs bred in Italy each year are enough only to cover $63 \%$ of the national needs, therefore, to meet its needs, Italy imports live pigs from other EU MSs. This constant flow of live pigs exposes Italy to the risk of importing and spreading infectious diseases.

Based on the available data and the desire of exploring the nature and the extent of contacts between pig holdings, in this work we aim at providing a preliminary description of the temporal pattern of pig movements recorded in Italy using data from a 4-year period (2013-2016), and identifying the areas or sectors at higher risk for the introduction and spread of infectious diseases transmitted by direct contact (e.g. African swine fever, classical swine fever, foot and mouth disease).

\section{2 | MATERIAL AND METHODS}

\section{1 | Source data}

Recording movement data are mandatory in Italy. Movement data are collected by the Italian National Livestock Registry (https:// www.vetinfo.sanita.it), covering the total of all pig movements. The Italian National Livestock registry provided batch-level data regarding every movement related to pig trade recorded from 1 January 2013 to 31 December 2016 on a daily basis. Each movement record reported the code of the holding of origin, the destination, the date of movement, and the number of pigs moved (batch). In addition, for each holding the Italian National Livestock registry provided the following attributes: type of holding (1. farm: farrowing/finishing/backyard, 2. staging point, i.e. to be slaughtered/ to be bred, 3. market, 4. assembly centre, 5 . genetic centre, i.e. genetic centre/quarantine centre/semen collection centre; 6 . slaughterhouse), type of production (1. farm: farrowing/finishing/ backyard), number of animals in the holdings on the day of the movement, geographical coordinates of the holding, municipality, province and region. For pigs coming imported into the country, the code of the holding of origin was replaced with the code of the Country of origin.

Additionally farms where classified in three classes (large, medium, small) on the basis of both the type of production and the number of pigs in the holding (large: farrowing $\geq 100$ heads or finishing $\geq 400$ heads, medium: farrowing $<100$ heads or finishing $<400$ heads, small: backyards, i.e. $<4$ heads).

\section{2 | Data analysis}

To identify whether seasonality was present, each year of the study period was divided in quarters, each representative of a season (winter: January, February, March; spring: April, May, June; summer: July, August, September; autumn: October, November, December), obtaining 16 time windows.

In this work, using the igraph $\mathrm{R}$ package, we built a directed network using holdings as nodes and pig movements as ties. After a preliminary description of the attributes of nodes and ties over the 
whole study period, basic network statistics as the average path length, the diameter, the shortest path and the degree distribution were obtained for each time window.

The temporal pattern of pig movements over the study period was then graphically described, obtaining for each $i$ of the 16 time windows the following parameters: (a) the number of nodes $\left(K_{i}\right),(b)$ the number of ties $\left(L_{i}\right)$, (c) the density $\left(D_{i}\right)$

$$
D_{i}=\frac{L_{i}}{K_{i\left(K_{i}-1\right)}} \epsilon[0,1]
$$

that is, the proportion of observed ties over the maximum possible number of ties as shown in Equation 1, (d) the number of pigs moved and (e) the number of imported pigs.

For each parameter, smoothing with annual moving averages was employed to explore if any long-term variation (trend) occurred. Moreover, to identify cyclical or irregular variations in pig movements specific and typical seasonal index were evaluated. The specific seasonal index highlights differences between the recorded frequency in a particular quarter and the expected value for that quarter, and it is obtained by subtracting the difference between the parameter value of a particular month and a 4-quarter smoothing average centred on that quarter. A typical seasonal index, calculated as the average of the specific seasonal indexes in different years, allows the identification of the seasonality of the phenomenon under study (Cowden, 1942).

Network communities (i.e. groups of highly connected nodes with few ties to other nodes) were then identified. Identifying and describing those communities are important in both forecasting the spread of diseases and in the resource allocation in surveillance (Relun et al., 2016).

Communities were identified using the walk-trap algorithm proposed by Pons and Latapy (2005), implemented in the igraph $R$ package.

We then described the highly populated clusters using the following 3 indices:

- the proportion of small farms that are part of the community

$$
\frac{n_{f, i}^{*}}{n_{f, i}}
$$

- the proportion of medium farms that are part of the community

$$
\frac{n_{m, i}^{*}}{n_{m, i}}
$$

- the proportion of large farms of the community

$$
\frac{n_{b, i}^{*}}{n_{b . i}}
$$

- the proportion of large farms of the community over the total elements of the community

Where $n_{f, i}$ is the total number of small farms at time $i, n_{m, i}$ is the total number of medium farms at time $i, n_{b, i}$ is the total number of large farms at time $i$. Whereas $n_{f, i}^{*}, n_{m, i}^{*}$ and $n_{b, i}$ are, respectively, the number of small farms, the number of medium farms and the number of large farms at time $i$ in the highly populated communities; finally, $n_{c, i}$ is the total number of farms in the highly populated communities at time $i$.

Then, we obtained the in-degree centrality, that is the number of in-going ties of each node $j$ in time $i$, as well as the out-degree centrality, that is the number of out-going ties of each node $j$ in time $i$.

Centrality is a measure of the 'importance' of nodes in a network, in terms of extension of their relationship with other nodes. Therefore, when dealing with infectious diseases, in-degree centrality can be considered a measure of the potential of a node of importing a disease, and the out-degree centrality can be considered a measure of the potential of a node in spreading a disease, allowing us to indicate nodes with the highest out-degree centrality as potential super-spreaders. Finally, we obtained the betweenness, a measure of the importance of a holding in connecting other holdings through being a part of their shortest path.

\section{RESULTS}

Our study involved a total of 91,475 holdings. As shown in Table 1, most of the holdings are farms (73\% small, $20 \%$ medium and $5 \%$ large).

Large farms are mostly located in Northern Italy (Pianura Padana), whereas medium and small farms are scattered over the whole country (data not shown). Overall, 1,853,081 movements were recorded during the study period. The highest number of animals was moved from farm to slaughterhouse (55\%) and from farm to farm (43\%). Approximately $2 \%$ of the pigs moved are imported from another EU MSs, mainly from Denmark (45\% of total movements from other EU MSs) and the Netherlands (15\%). Table 2 shows a description of the batch size of ingoing and outgoing movements, by the type of holding. Import movements from other countries, in general, have the largest outgoing batch size, whereas movements from finishing farms have the largest ingoing batch size.

$84 \%$ of imported pigs was introduced into large finishing farms located in Northern Italy (Figure 1).

The number of introduced pigs, mean batch size and interquartile range of the batch size by Country of origin and type of holding of destination is reported in table A.3, in Supplementary Material. The basic network statistics (average path length, shortest path, diameter) for each time window are reported in table A.4, in 


\begin{tabular}{|c|c|c|c|c|}
\hline Type of holding & $\begin{array}{l}\text { Type of } \\
\text { production }\end{array}$ & Size & $\begin{array}{l}\text { Frequency (Number } \\
\text { of holdings) }\end{array}$ & Percent \\
\hline Farm & Backyard & Small farm & 67,112 & $73.37 \%$ \\
\hline Farm & Farrowing & Medium farm & 9,388 & $10.26 \%$ \\
\hline Farm & Finishing & Medium farm & 8,831 & $9.65 \%$ \\
\hline Farm & Finishing & Large farm & 2,998 & $3.28 \%$ \\
\hline Farm & Farrowing & Large farm & 1,491 & $1.63 \%$ \\
\hline Slaughterhouse & & & 1,289 & $1.41 \%$ \\
\hline Farm & \multicolumn{2}{|l|}{ Uncategorized } & 130 & $0.14 \%$ \\
\hline Market & & & 101 & $0.11 \%$ \\
\hline Staging point & \multicolumn{2}{|l|}{ To be bred } & 50 & $0.05 \%$ \\
\hline Staging point & \multicolumn{2}{|l|}{$\begin{array}{l}\text { To be } \\
\text { slaughtered }\end{array}$} & 35 & $0.04 \%$ \\
\hline Genetic centre & \multicolumn{2}{|l|}{ Genetic centre } & 14 & $0.02 \%$ \\
\hline Genetic centre & \multicolumn{2}{|c|}{ Semen collection centre } & 11 & $0.01 \%$ \\
\hline Genetic centre & \multicolumn{2}{|l|}{$\begin{array}{l}\text { Quarantine } \\
\text { centre }\end{array}$} & 10 & $0.01 \%$ \\
\hline \multicolumn{3}{|l|}{ Assembly centre } & 8 & $0.01 \%$ \\
\hline Staging point & \multicolumn{2}{|l|}{ Uncategorized } & 7 & $0.01 \%$ \\
\hline Total & & & 91,475 & \\
\hline
\end{tabular}

TABLE 1 Description of the holdings entered in the study, by type of holding, type of production and size (large: farrowing $\geq 100$ heads or finishing $\geq 400$ heads, medium: farrowing $<100$ heads or finishing $<400$ heads, small: backyards, i.e. $<4$ heads)
Supplementary Material. All those statistics are constant during the whole study period with values ranging between 2 and 5 for the average path length, between 5 and 6 for the shortest path length and between 8 and 14 for the diameter.

The number of nodes active season by season shows a seasonal pattern, with a decreasing trend across the whole study period. The typical and the specific seasonal indexes show an increase in the number of active nodes in spring (Figures 2, 3 and Figure A5 in Supplementary Material (a)). The number of ties also, shows a seasonal pattern, but without trend across the study period. The typical and the specific seasonal indexes show an increase in the number of ties in winter and autumn (Figures 2, 3 and Figure A5 in Supplementary Material (b)). The density, that is the number of ties over the maximum possible ties, shows a seasonal pattern, with an increasing trend. The typical and the specific seasonal indexes show an increase in density in winter and autumn (Figures 2, 3 and Figure A5 in Supplementary Material (c)). Finally, the number of pigs moved shows 2 peaks during the autumn of 2014 and the spring of 2016, with an increasing trend in the number of pigs moved. The typical and the specific seasonal indexes show an increase in number of the pigs moved in spring and winter (Figures 2, 3 and Figure A5 in Supplementary Material (d)). The number of imported pigs also shows seasonality and an abrupt increasing trend (Figure A6 in Supplementary Material).

The analysis for the identification of communities, showed the existence of one highly populated community (about 3,000 nodes), 2 to 6 less populated clusters (300-500 nodes) and several smaller communities. The total number of communities identified ranges between nearly 1,200 and 1,800 (mean 1,449, standard deviation 159.48), depending on the time period (Figures a.10-a.13, in Supplementary Material). The highly populated community shows the same location over the whole study time period, mainly in Northern Italy, in correspondence with the area of the Pianura Padana.

The less populated communities also are generally located in well-defined areas (Piemonte, Veneto, Trentino Alto Adige, Campania, Umbria) during the whole study period, whereas small communities are scattered across the whole country (Figure 4, Figures a.10-a.13 in Supplementary Material). A clear seasonality of the number of active nodes emerges when analysing the number of active nodes belonging to the communities.

A statistically significant decreasing trend in the number of active nodes is evident only when considering nodes represented by small farms (Figure 5a-c). The number of total active nodes in the highly populated community, shown in Figure 5 (d), shows an abrupt decreasing trend over the study period.

The proportion of active nodes represented by either small or medium or large farms belonging to the highly populated community, over the total number of active nodes representing the respective size of farms, shows an abrupt statistically significant decreasing trend in nodes representing medium farms and a decreasing trend in nodes representing large farms (Figure 6a-c). A clear statistically significant increasing trend was found analysing the proportion of nodes representing large farms in the highly populated community over the total number of nodes in the highly populated community (Figure 6d).

The location of nodes with high in-degree and nodes with high out-degree (i.e. potential super-spreaders) is constant during the whole study period and, as shown in Figure 7, most of them are located in Pianura Padana. Conversely, the in-degree and the 
TABLE 2 Batch size of ingoing and outgoing movements by type of holding. Countries are identified by their ISO 3166-1 Alpha2 codes

\begin{tabular}{|c|c|c|c|c|}
\hline \multirow[b]{2}{*}{ Type of holding } & \multicolumn{2}{|c|}{ Outgoing movement } & \multicolumn{2}{|c|}{ Ingoing movement } \\
\hline & Median & $\begin{array}{l}\text { Interquartile } \\
\text { range }\end{array}$ & Median & $\begin{array}{l}\text { Interquartile } \\
\text { range }\end{array}$ \\
\hline Assembly centre & 30 & 55 & 14 & 32 \\
\hline Farm:backyard & 2 & 2 & 2 & 0 \\
\hline Farm:farrowing & 9 & 64 & 20 & 107 \\
\hline Farm:finishing & 10 & 128 & 246 & 505 \\
\hline Farms:unspecified & 2 & 1 & 3 & 10 \\
\hline Genetic centre & 10 & 30 & 4 & 5 \\
\hline Market & 2 & 3 & 11 & 13 \\
\hline Slaughterhouse & - & - & 9 & 69 \\
\hline Staging point & 4 & 18 & 30 & 60 \\
\hline Foreign country (all) & 344 & 494 & 95 & 168 \\
\hline$A L$ & 117 & 0 & - & - \\
\hline AT & 2 & 449 & 10 & 17 \\
\hline BE & 56 & 109 & 173 & 160 \\
\hline CA & - & - & 15 & 8 \\
\hline $\mathrm{CH}$ & - & - & 12 & 0 \\
\hline$C Z$ & - & - & 150 & 1,013 \\
\hline DE & 108 & 790 & 181 & 320 \\
\hline DK & 68 & 0 & 630 & 357 \\
\hline ER & 7 & 6 & - & - \\
\hline ES & 182 & 90 & 190 & 170 \\
\hline FR & - & - & 150 & 130 \\
\hline HR & - & - & 160 & 50 \\
\hline $\mathrm{HU}$ & 178 & 94 & 180 & 45 \\
\hline IE & - & - & 28 & 35 \\
\hline LU & - & - & 185 & 10 \\
\hline MT & - & - & 80 & 55 \\
\hline NL & 83 & 0 & 521 & 645 \\
\hline $\mathrm{PL}$ & - & - & 160 & 152 \\
\hline SI & 2 & 1 & 8 & 9 \\
\hline SK & 200 & 0 & 750 & 50 \\
\hline SM & 7 & 11 & - & - \\
\hline UK & - & - & 175 & 191 \\
\hline UY & 90 & 0 & - & - \\
\hline
\end{tabular}

out-degree show a seasonal pattern superimposable to the pattern seen in the network description (Figure 8). Between the in-degree and out-degree of nodes, there is a correlation ranging from -0.004 to -0.029 , depending on the study period. This indicates of the presence of disassortativeness in the connections. Highly central nodes in term of their outgoing movement do not tend to be connected to highly central nodes in terms of ingoing movements. This property is important for disease spread since nodes, which are likely to get a disease from ingoing movements, do not have also high chance of further transmitting the disease.

Finally, the betweenness values for the nodes with higher degree mean values are relatively high (data not shown), meaning that those nodes are in the shortest path between two other nodes, and consequently, if infected, they can spread the disease.

\section{DISCUSSION}

With our study, we described specific seasonal patterns of pig movements in Italy, we highlighted the Pianura Padana (Northern Italy) as the area at highest risk for the introduction and the spread of pig infectious diseases, and finally we identified nodes throughout the country as potential locations for super-spreaders. The data we have made available will be useful to the study of the spread of swine 


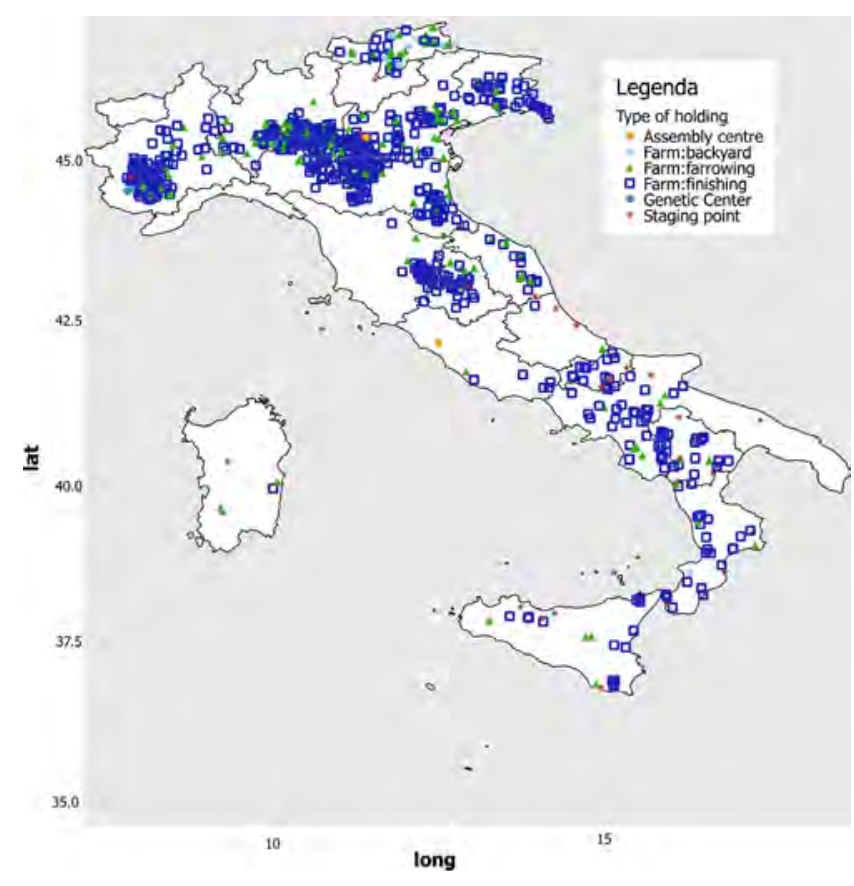

FIGURE 1 Destination of pigs imported from other EU Countries to Italy during the whole study period (2013-2016), by type of holding of destination. Long = longitude, lat $=$ latitude

diseases and to the subsequent implementation of risk-based surveillance plans.

Aiming at being as general as possible, we did not apply any simulation technique for any specific disease, so we are aware that for each single pathogen our study should be improved by simulation, for example implementing susceptible-infectious-recoverd (SIR) compartmental models, taking into account its specificity in terms of disease spread and animal movements, as suggested by Bajardi et al. (2012) in cattle.

Our study was based on official data regarding all pig movements in Italy between 1 January 2013 and 31 December 2016 provided by the National Livestock database, an institution of the Italian Ministry of Health, able to ensure that that reporting bias is minimized. A previous description of pig movements in Italy was provided by Relun et al. (2016), comparing the spatial and functional organization of the pig trade in 2011 in 4 EU MSs. In this work, the walk-trap algorithm was able to identify an intensive production system in northern Italy, as well as small scale pig production systems in central and southern Italy. Applying the same method to a longer series of data, we were able to better characterize both the intensive production system, represented by the large farms and the smaller production cycle, represented by the medium and small farms.

The network we built shows a scale-free structure, similar to what is reported in literature for the bigger pig producers in Europe (Rautureau et al., 2011; Relun et al., 2016). Theoretical studies have shown that the scale-free (SF) structure can influence the spread and the extent of a disease (Kiss et al., 2006a; Pastor-Satorras \& Vespignani, 2001), their highly skewed degree distribution results in a basic reproduction number that can be very high, even when the transmission probability is low.

Risk-based surveillance plans also need to consider the seasonality in pig movements to be most effective. Seasonality was found in the number of active nodes over time in both the intensive and in the smaller production system: this result is in contrast with other EU MSs, where seasonality was not found or found only in the small producing sector (Porphyre et al., 2014; Salines et al., 2017).

The overall number of holdings is decreasing overtime, due to the decline in the number of small farms, in agreement with the general trend observed by another EU pig-producing MS (Guinat et al., 2016; Marquer and Teresa Rabade, 2014).

Connections among farms show an opposite increasingly trend: pig farms are becoming increasingly large and connected a wellknown tendency of the Italian pig industry.

Data were analysed using SNA techniques previously applied to model pattern of disease transmission among livestock in several countries. A potential limitation of our study is that the presented analysis can be referred to diseases that spread through direct contact (e.g. Classical Swine Fever) as there are gaps in the data that do not allow to apply the same techniques to transmission via fomites or transmission via vehicles or people movements. To our knowledge, most studies in literature are focused on giving a static time frame of pig movements, and only a few explored the temporal pattern of pig movements (Guinat et al., 2016; Lentz et al., 2016; MartinezLopez et al., 2009a; Noremark et al., 2011; Porphyre et al., 2014). The primary focus of our study, however, was to describe how pig movements vary over time. As we wanted to evaluate seasonality, if present, a data aggregation on a 3-month time window was considered appropriate. A clear seasonal pattern of all the parameters evaluated emerged, revealing a higher number of movements of pig batches in spring and autumn, linked with the breeding and production cycle as pigs moved from the growing to the finishing phase and with periods of increased slaughtering, particularly before Christmas and Easter. This seasonal pattern has also been described in another EU MS (Guinat et al., 2016; Martinez-Lopez et al., 2009a; Noremark et al., 2011; Porphyre et al., 2014). The same seasonal pattern has been found when restricting the analysis to the movements of imported pig batches. Outbreaks occurring during the periods of increased movement of pig batches would have a greater impact in the spread of an infectious disease; therefore, an additional effort in surveillance during those periods may be appropriate. Also, the increasing trend in the number of pigs imported from other countries highlights an increasing risk of importing pig infectious diseases exotic to Italy and therefore the need for an enhanced surveillance.

In our model, the destination of imported pigs was in line with pig density; therefore, pig density, a parameter that is widely available and easy to use as an input in risk assessment models, was proven to be a suitable proxy for where the pigs enter the countries.

We were able to detect a highly populated community located in northern Italy, an area known to be the centre of the Italian industrial pig production. This area (Pianura Padana) also receives most of the pigs imported to Italy. 
(a)

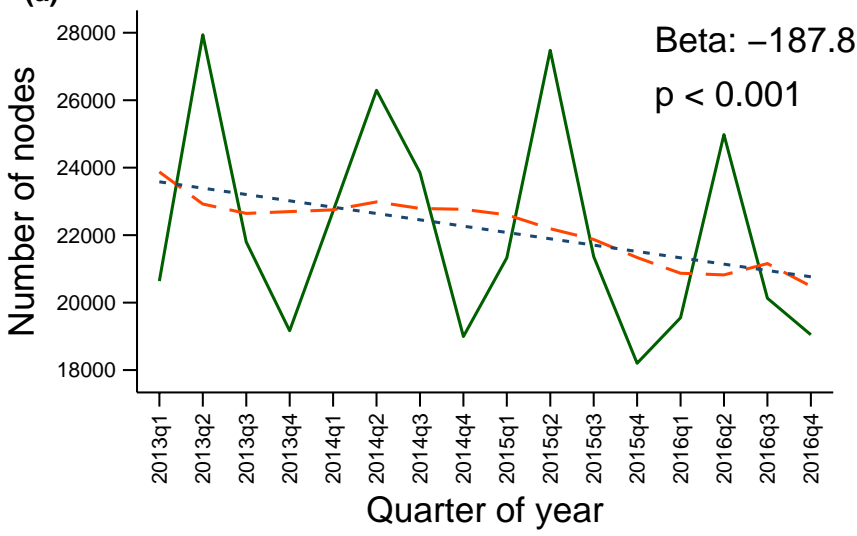

(c)

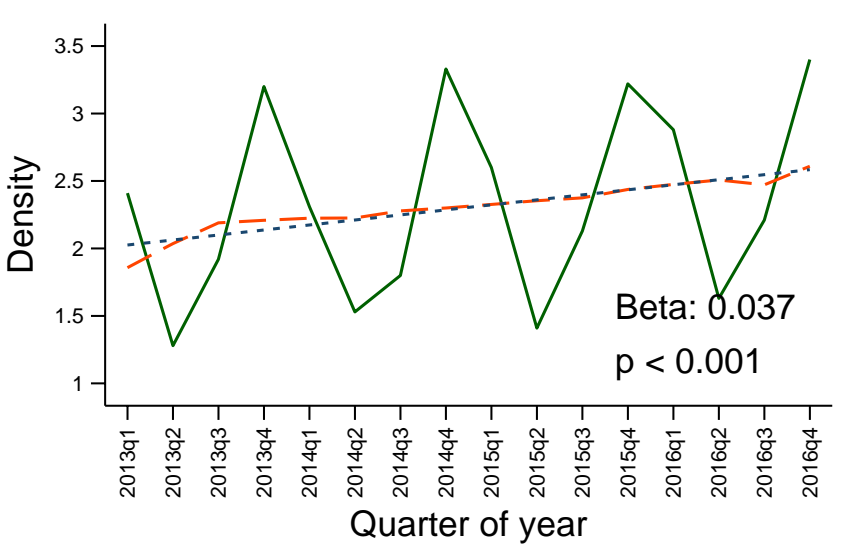

(b)

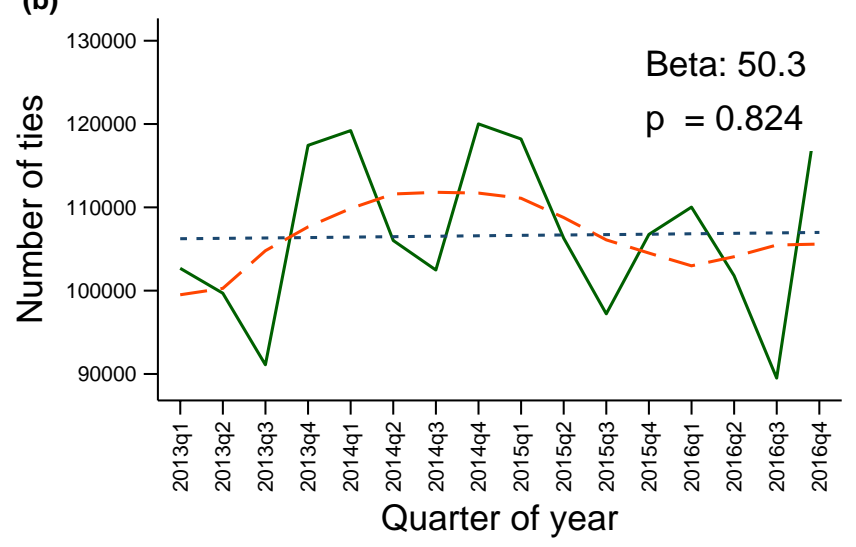

(d)

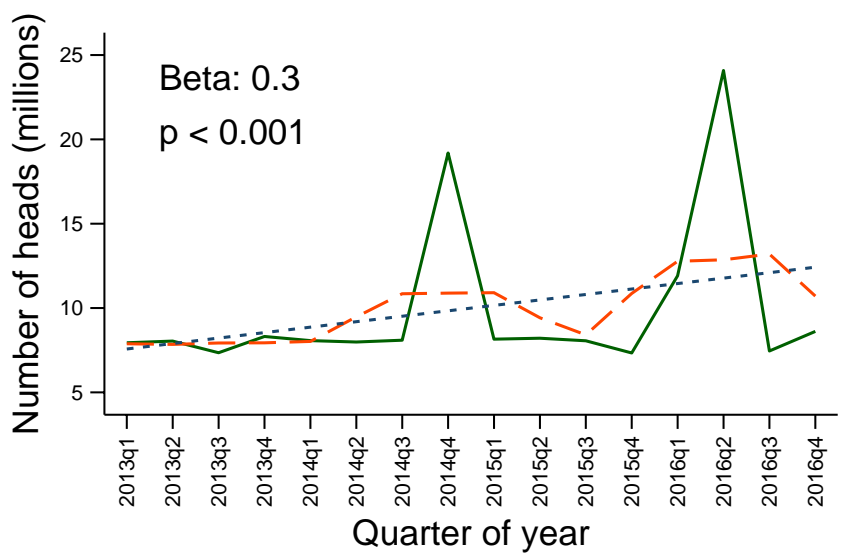

FIGURE 2 Descriptive statistics of temporal pattern of pigs movements in the period 2013-2106: (a) Raw count (solid line), annual moving averages (dashed line) and linear trend (dotted line) of the number of nodes; (b) Raw count (solid line), annual moving averages (dashed line) and linear trend (dotted line) of the number of ties; (c) Raw quantification (solid line), annual moving averages (dashed line) and linear trend (dotted line) of density, (d) Raw count (solid line), annual moving averages (dashed line) and linear trend (dotted line) of number of pigs (expressed in millions)

FIGURE 3 Seasonal typical index of pigs movements in the period 2013-2106: (a) number of nodes; (b) number of ties; (c) density and (d) number of pigs (expressed in millions)

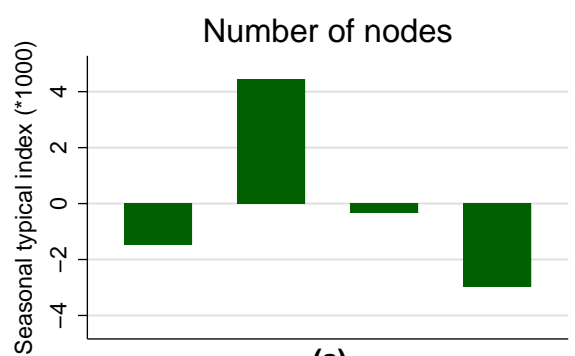

(a)

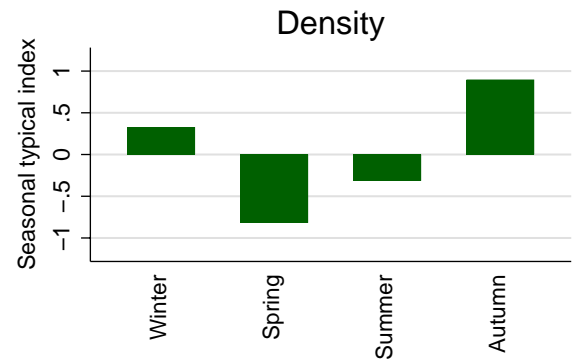

(c)

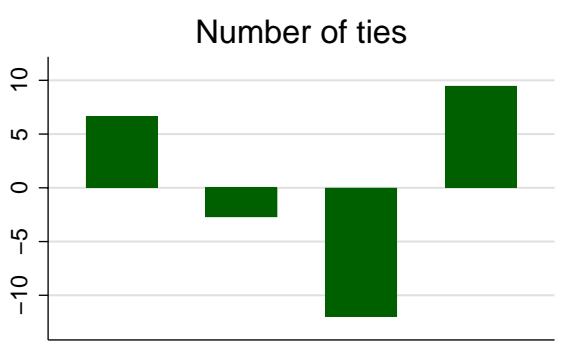

(b)

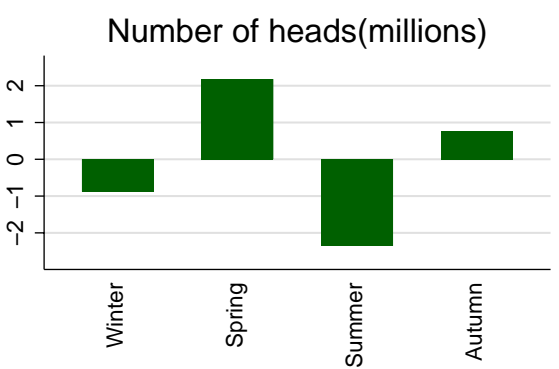

(d) 

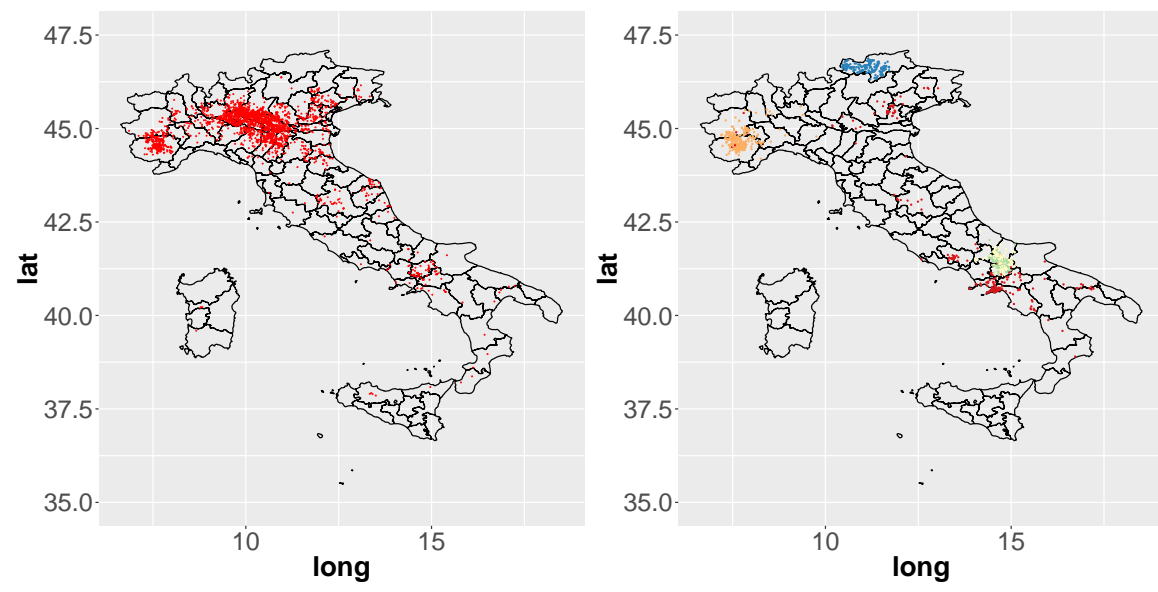

FIGURE 4 Community description. (a) Location of the nodes that belong to the highly populated community. (b) Location of the nodes belonging to the 2-6 top populated communities in the second spring in the study (second quarter of 2014). Long = longitude, lat $=$ latitude

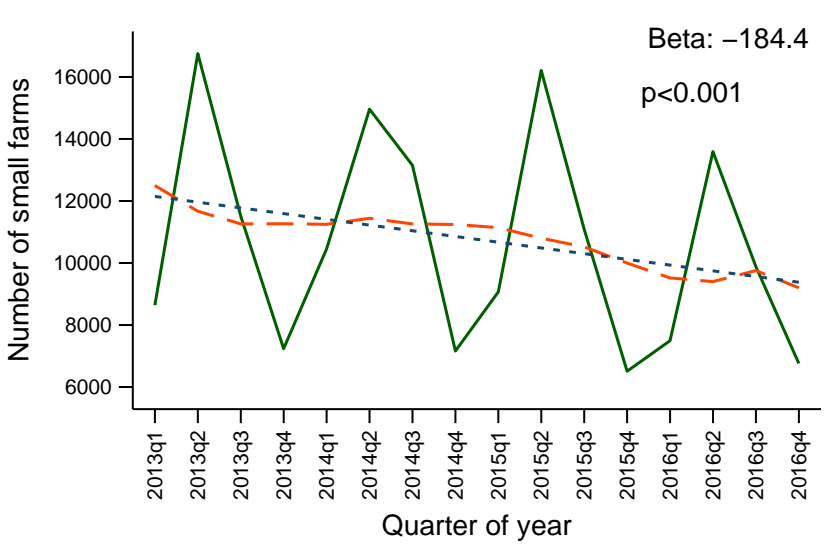

(a)

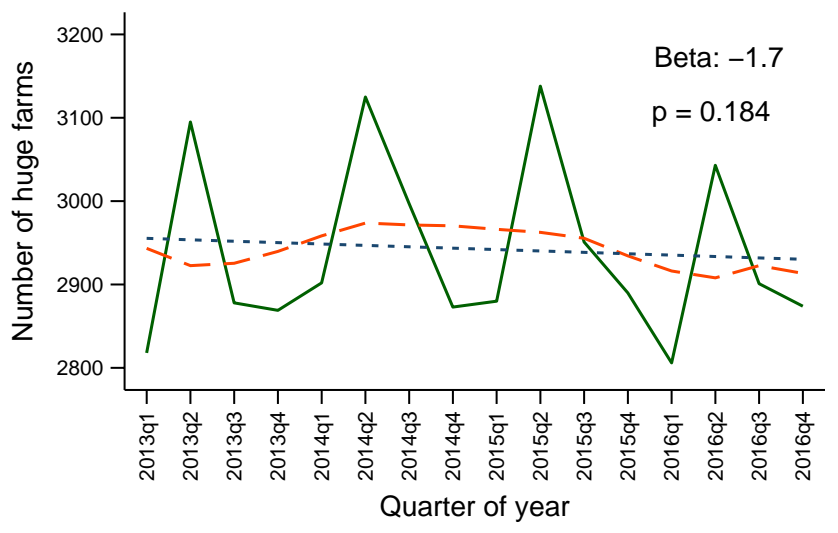

(c)

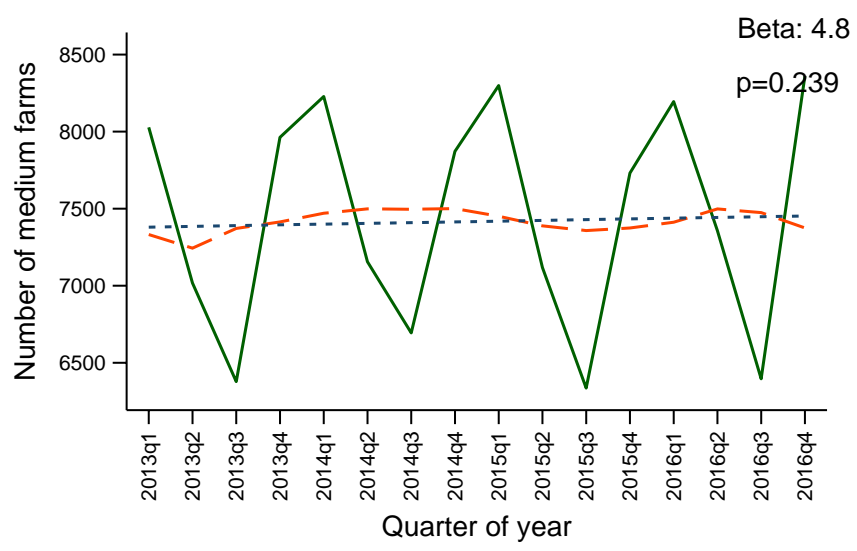

(b)

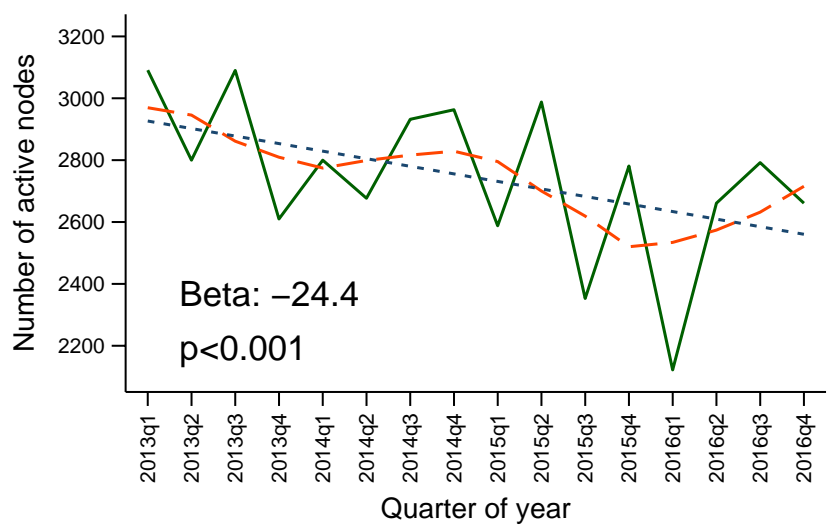

(d)

FIGURE 5 Description of active nodes in communities:(a) total number of nodes representing small farms, (b) total number of nodes representing medium farms, (c) total number of nodes representing large farms and (d) total number of active nodes in the highly populated community. Solid line: raw data, dashed line: annual moving averages, dotted line: linear trend

This result confirms the potential role of Pianura Padana in the spread of infectious diseases in pigs. The elements of the highly populated community are not only large farms ( $55 \%$ of the farms in the community), but also medium and small size farm. Assuming that farms of different size have different biosecurity levels, those connections between holdings with different biosecurity levels will deserve particular consideration when evaluating the potential spread of an infectious disease. The walk-trap algorithm, that allowed us to identify the communities, is typically used for static networks. We applied it to a series of static time windows; therefore, the communities they detect 


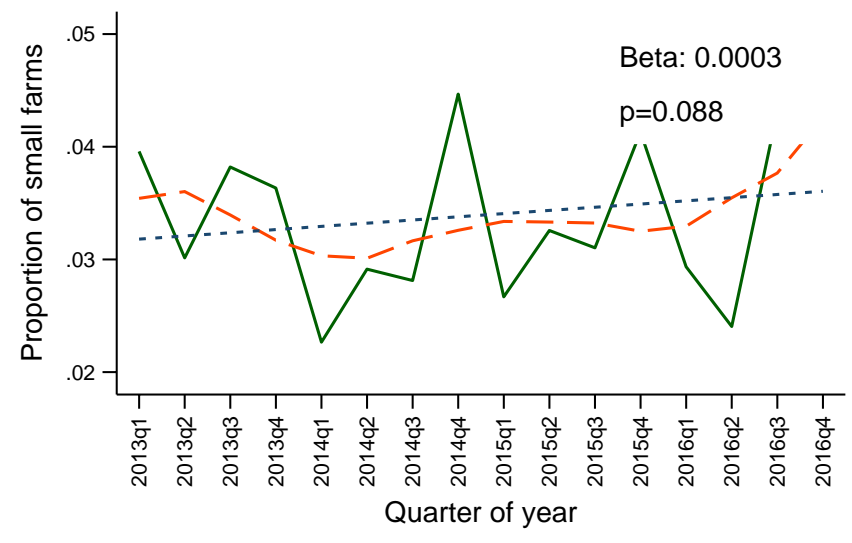

(a)

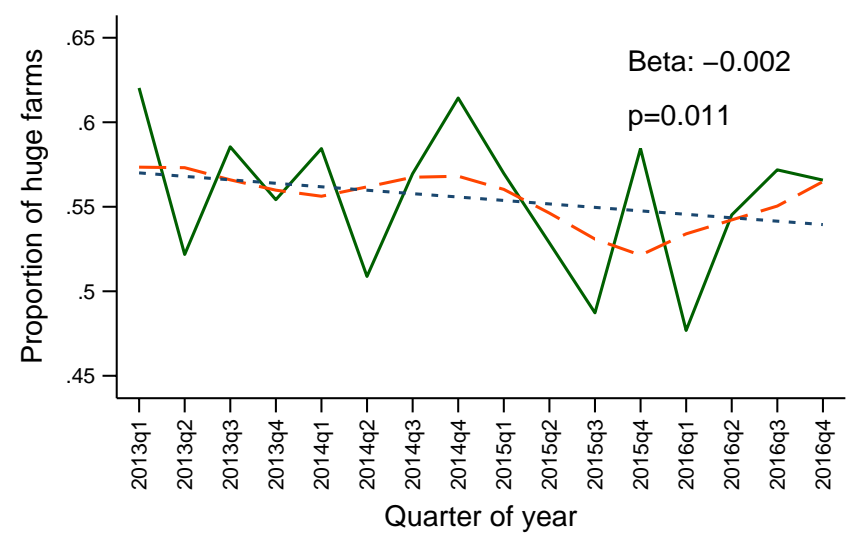

(c)

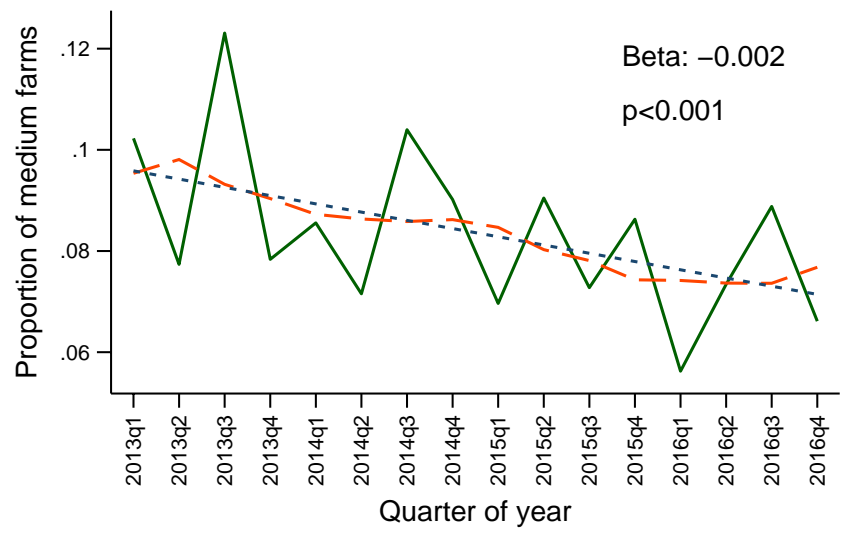

(b)

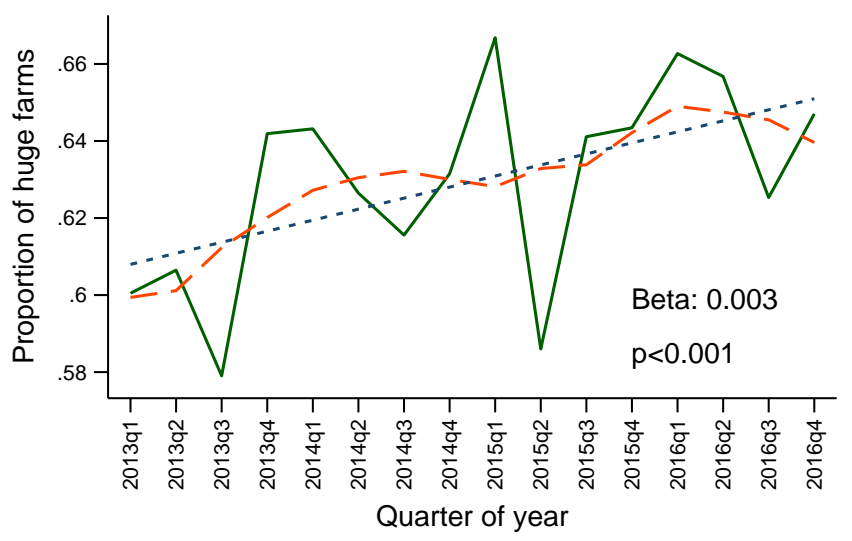

(d)

FIGURE 6 Community description. (a) proportion of active nodes representing small farms in the highly populated community over the total number of active nodes representing small farms, (b) proportion of active nodes representing medium farms in the highly populated cluster over the total number of active nodes representing medium farms, (c) proportion of active nodes representing large farms in the highly populated community over the total number of active nodes representing large farms and (d) proportion of active nodes representing large farms in the highly populated community over the total number of active nodes in the highly populated community. Solid line: raw data, dashed line: annual moving averages, dotted line: linear trend

FIGURE 7 Potential super-spreaders, that is nodes with high out-degree centrality (a) and high in-degree centrality (b). Bullets are proportional to the mean degree centrality. Long = longitude, lat $=$ latitude (a)

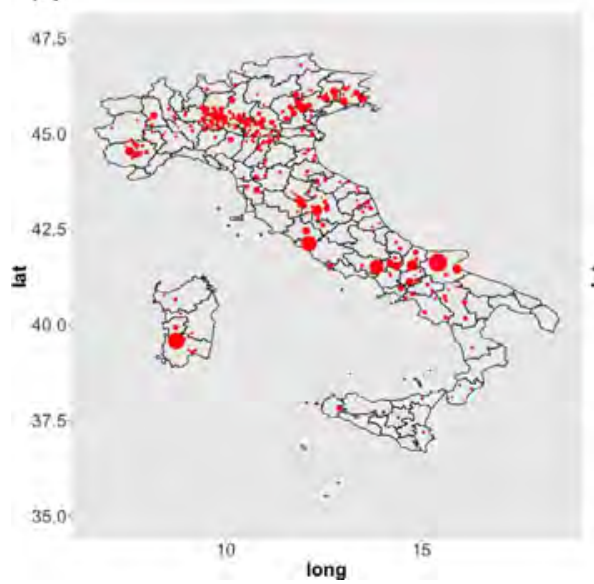

(b)

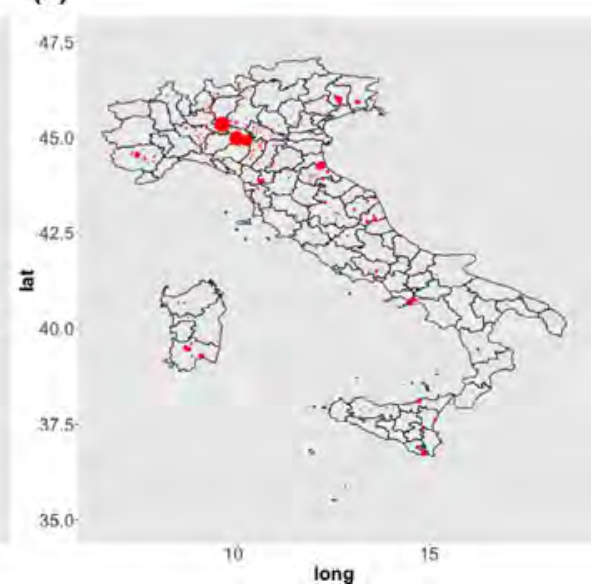

in each time window are the accurate reflection of the real communities only in that time window. However, since the spatial pattern of those communities was constant over time, it seems reasonable that the detected communities overlap each time.
SNA allowed us to identify some nodes as potential super-spreaders. Those nodes are constantly present during the whole study period with a similar ranking in their potential of being super-spreaders. The recurring seasonal pattern of the network 


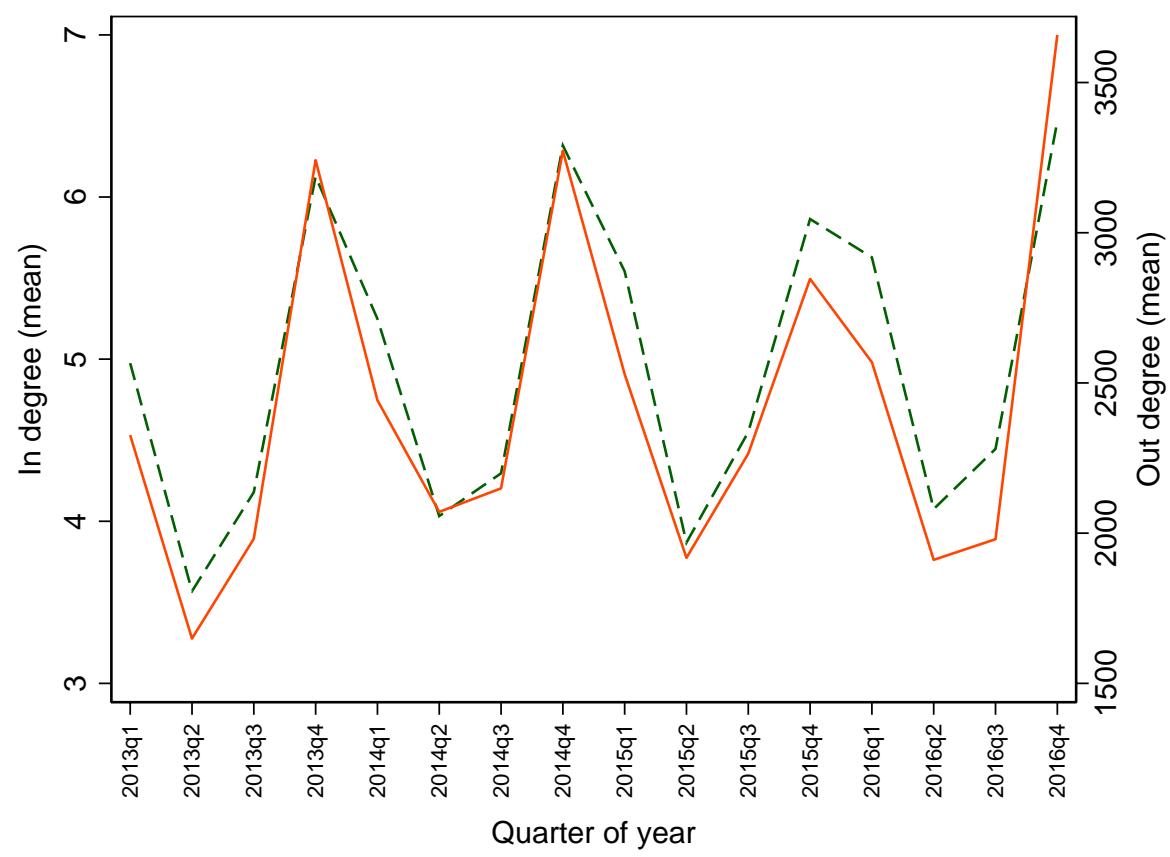

FIGURE 8 Centrality description. Mean of in-degree (dashed line) and out-degree (solid line) in the study period is displayed also by in-degee and out-degree, whereas the spatial distribution of the nodes with high in-degree and out-degree is constant during the time. The relatively high betweenness values of super-spreaders allow us to suppose that they have a potential role in the spreading on the infection, being on the shortest path connecting two nodes. Moreover, our network show disassortativeness, suggesting that there are central farms (hubs) that are the most likely to spread a disease and that could be targeted by control measures.

Most of super-spreaders are located in Northern Italy, but, notably, super-spreaders with high mean in and out-degree centrality are located also in Sardegna, Lazio, Puglia and Campania.

In conclusion when planning surveillance activities and when applying disease control strategies to the pig trade in Italy the following elements should be considered: the seasonality of the network, the presence of communities located mostly in Pianura Padana and the presence of super-spreaders located not only in northern Italy, but also in other regions.

\section{ACKNOWLEDGEMENTS}

The authors gratefully acknowledge the Italian National Livestock Registry (https://www.vetinfo.sanita.it) for provision of the data.

\section{CONFLICT OF INTEREST}

The authors report no conflict of interest.

\section{ETHICAL APPROVAL}

The authors confirm that the ethical policies of the journal, as noted on the journal's author guidelines page, have been adhered to. No ethical approval was required as the data used in this research are routinely and mandatorily collected for surveillance purposes.

\section{DATA AVAILABILITY STATEMENT}

The data that support the findings of this study are available from the Italian Ministry of Health. Restrictions apply to the availability of these data, which were used under license for this study. Data are available at https://www.vetinfo.it/ with the permission of The Ministry of Health.

\section{ORCID}

Maria Ines Crescio iD https://orcid.org/0000-0002-6761-3528

Marco DeNardi iD https://orcid.org/0000-0001-5648-257X

\section{REFERENCES}

Bajardi, P., Barrat, A., Natale, F., Savini, L., \& Colizza, V. (2011). Dynamical patterns of cattle trade movements. PLoS One, 6, e19869. https://doi. org/10.1371/journal.pone.0019869

Bajardi, P., Barrat, A., Savini, L., \& Colizza, V.(2012). Optimizing surveillance for livestock disease spreading through animal movements. Journal of the Royal Society, Interface, 76, 2814-2825. https://doi. org/10.1098/rsif.2012.0289

Bigras-Poulin, M., Thompson, A., Chriel, M., Mortensen, S., Greiner, M., Reid, S., Menzies, F., \& Russell, A. (2004). Network analysis of the Danish cattle and swine industry trade patterns as an evaluation of risk potential for disease spread: The heterogeneity issue. In: Society for veterinary epidemiology and preventive medicine (pp. 189-199). : Proceedings of a meeting held at the Martigny, Switzerland on the 24th-26th March; 2004.

Brennan, M. L., Kemp, R., \& Christley, R. M. (2008). Direct and indirect contacts between cattle farms in north-west England. Preventive Veterinary Medicine, 84, 242-260. https://doi.org/10.1016/j.preve tmed.2007.12.009

Brioudes, A., \& Gummow, B. (2017). Understanding pig and poultry trade networks and farming practices within the pacific islands as a basis for surveillance. Transboundary and Emerging Diseases, 64, 284-299. https://doi.org/10.1111/tbed.12370

Buttner, K., Krieter, J., Traulsen, A., \& Traulsen, I. (2013). Static network analysis of a pork supply chain in Northern Germanycharacterisation of the potential spread of infectious diseases via 
animal movements. Preventive Veterinary Medicine, 110, 418-428. https://doi.org/10.1016/j.prevetmed.2013.01.008

Cowden, D. J. (1942). Moving seasonal indexes. Journal of the American Statistical Association, 220, 523-524. https://doi.org/10.1080/01621 459.1942.10500654

Dorjee, S., Revie, C., Poljak, Z., McNab, W., \& Sanchez, J. (2013). Network analysis of swine shipments in Ontario, Canada, to support disease spread modelling and risk-based disease management. Preventive Veterinary Medicine, 112, 118-127. https://doi.org/10.1016/j.preve tmed.2013.06.008

Dube, C., Ribble, C., Kelton, D., \& McNab, B. (2008). Comparing network analysis measures to determine potential epidemic size of highly contagious exotic diseases in fragmented monthly networks of dairy cattle movements in Ontario, Canada. Transboundary and Emerging Diseases, 55, 382-392. https://doi.org/10.1111/j.1865-1682.2008.01053.x

Dube, C., Ribble, C., Kelton, D., \& McNab, B. (2011). Introduction to network analysis and its implications for animal disease modelling. Revue Scientifique et Technique de I'OIE, 30, 425-436. https://doi. org/10.20506/rst.30.2.2043

Frossling, J., Ohlson, A., Bjorkman, C., Haakansson, N., \& Noremark, M. (2012). Application of network analysis parameters in risk-based surveillance examples based on cattle trade data and bovine infections in Sweden. Preventive Veterinary Medicine, 3, 202-208. https://doi. org/10.1016/j.prevetmed.2011.12.011

Guinat, C., Relun, A., Wall, B., Morris, A., Dixon, L., \& Pfeiffer, D. U. (2016). Exploring pig trade patterns to inform the design of riskbased disease surveillance and control strategies. Scientific Reports, 6, 28429. https://doi.org/10.1038/srep28429

Kiss, I. Z., Green, D. M., \& Kao, R. R. (2006a). Infectious disease control using contact tracing in random and scale-free networks. Journal of the Royal Society, Interface, 3, 669-677. https://doi.org/10.1098/ rsif.2005.0079

Kiss, I. Z., Green, D. M., \& Kao, R. R. (2006b). The network of sheep movements within Great Britain: Network properties and their implications for infectious disease spread. Journal of the Royal Society Interface, 10, 669-677. https://doi.org/10.1098/rsif.2006.0129

Lentz, H. H., Koher, A., Hovel, P., Gethmann, J., Sauter-Louis, C., Selhorst, T., \& Conraths, F. J. (2016). Disease spread through animal movements: A static and temporal network analysis of pig trade in Germany. PLoS One, 11, e0155196. https://doi.org/10.1371/journal.pone.0155196

Lentz, H., Konschake, M., Teske, K., Kasper, M., Rother, B., Carmanns, R., Petersen, B., Conraths, F., \& Selhorst, T. (2011). Trade communities and their spatial patterns in the German pork production network. Preventive Veterinary Medicine, 98(332), 176-181. https://doi. org/10.1016/j.prevetmed.2010.10.011

Lindstrom, T., Lewerin, S. S., \& Wennergren, U. (2012). Influence on disease spread dynamics of herd characteristics in a structured livestock industry. Journal of the Royal Society, Interface, 71, 1287-1294. https://doi.org/10.1098/rsif.2011.0625

Marquer, P., \& Teresa Rabade, R. F. (2014). Pig farming in the European Union: Considerable variations from one member state to another. https://ec.europa.eu/eurostat/statistics-explained/index.php?title =Archive:Pig_farming_sector_-_statistical_portrait_2014.

Martinez-Lopez, B., Perez, A., \& Sanchez-Vizcaino, J. (2009a). Combined application of social network and cluster detection analyses for temporal-spatial characterization of animal movements in Salamanca, Spain. Preventive Veterinary Medicine, 91, 29-38. https://doi. org/10.1016/j.prevetmed.2009.05.007

Martinez-Lopez, B., Perez, A. M., \& Sanchez-Vizcaino, J. M. (2009b) Social network analysis. Review of general concepts and use in preventive veterinary medicine. Transboundary and Emerging Diseases, 56, 109-120. https://doi.org/10.1111/j.1865-1682.2009.01073.x

Natale, F., Giovannini, A., Savini, L., Palma, D., Possenti, L., Fiore, G., \& Calistri, P. (2009). Network analysis of Italian cattle trade patterns and evaluation of risks for potential disease spread. Preventive
Veterinary Medicine, 92, 341-350. https://doi.org/10.1016/j.preve tmed.2009.08.026

Noremark, M., Haakansson, N., Lewerin, S. S., Lindberg, A., \& Jonsson, A. (2011). Network analysis of cattle and pig movements in Sweden: Measures relevant for disease control and risk-based surveillance. Preventive Veterinary Medicine, 99, 78-90. https://doi.org/10.1016/j. prevetmed.2010.12.009

Pastor-Satorras, R., \& Vespignani, A. (2001). Epidemic spreading in scale-freenetworks. Physical Review Letters, 86, 3200-3203. https:// doi.org/10.1103/PhysRevLett.86.3200

Pons, P., \& Latapy, M. (2005). Computing communities in large networks using random walks. In P. Yolum, T. Güngör, F. Gürgen, \& C. Özturan (Eds.), Computer and information sciences - ISCIS 2005 (pp. 284-293). Springer.

Porphyre, T., Boden, L. A., Correia-Gomes, C., Auty, H. K., Gunn, G. J., \& Woolhouse, M. E. (2014). How commercial and non-commercial swine producers move pigs in Scotland: A detailed descriptive analysis. BMC Veterinary Research, 10(1), 10-140. https://doi. org/10.1186/1746-6148-10-140

Rautureau, S., Dufour, B., \& Durand, B. (2011). Vulnerability of animal trade networks to the spread of infectious diseases: A methodological approach applied to evaluation and emergency control strategies in cattle, France. 2005. Transboundary and Emerging Diseases, 58, 110-120. https://doi.org/10.1111/j.1865-1682.2010.01187.x

Relun, A., Grosbois, V., Sanchez-Vizcaino, J. M., Alexandrov, T., Feliziani, F., Waret-Szkuta, A., Molia, S., Etter, E. M., \& Martinez-Lopez, B. (2016). Spatial and functional organization of pig trade in different European production systems: Implications for disease prevention and control. Frontiers in Veterinary Science, 3, 4. https://doi. org/10.3389/fvets.2016.00004

Ribbens, S., Dewulf, J., Koenen, F., Mintiens, K., de Kruif, A., \& Maes, D. (2009). Type and frequency of contacts between Belgian pig herds. Preventive Veterinary Medicine, 88, 57-66. https://doi.org/10.1016/j. prevetmed.2008.08.002

Salines, M., Andraud, M., \& Rose, N. (2017). Pig movements in France: Designing network models fitting the transmission route of pathogens.2017. PLoS One, 12, e0185858. https://doi.org/10.1371/journ al.pone. 0185858

Thakur, K. K., Revie, C. W., Hurnik, D., Poljak, Z., \& Sanchez, J. (2016). Analysis of swine movement in four Canadian regions: Network structure and implications for disease spread. Transboundary and Emerging Diseases, 63, e14-e26. https://doi.org/10.1111/tbed.12225

Vernon, M. C., \& Keeling, M. J. (2009). Representing the UK's cattle herd as static and dynamic networks. Proceedings of the Royal Society B: Biological Sciences, 276(1656), 469-476. https://doi.org/10.1098/ rspb.2008.1009

Volkova, V. V., Howey, R., Savill, N. J., \& Woolhouse, M. E. (2010). Sheep movement networks and the transmission of infectious diseases. PLoS One, 5, e11185. https://doi.org/10.1371/journal.pone. 0011185

\section{SUPPORTING INFORMATION}

Additional supporting information may be found online in the Supporting Information section.

How to cite this article: Crescio MI, Mastrantonio G, Bertolini S, et al. Using network analysis to identify seasonal patterns and key nodes for risk-based surveillance of pig diseases in Italy. Transbound Emerg Dis. 2020;00:1-11. https://doi.org/10.1111/tbed.13960 